\title{
The Circulation Distribution on the Lifting Line for a Given Extracted Power
}

\author{
Ali Helali, ${ }^{1}$ Badreddine Kamoun, ${ }^{2}$ David Afungchui, ${ }^{3}$ and Mohamed Jomaa Safi ${ }^{1}$ \\ ${ }^{1}$ Research Unit of Mechanics and Energetic (URME), National Engineering School of Tunis, 1002 Tunis, Tunisia \\ ${ }^{2}$ Laboratory of Applied Physics (LPA), Faculty of Sciences of Sfax, 3038 Sfax, Tunisia \\ ${ }^{3}$ Department of Physics, Faculty of Sciences, University of Buea, Buea, Cameroon \\ Correspondence should be addressed to Ali Helali, helaliali@voila.fr
}

Received 28 April 2011; Revised 1 August 2011; Accepted 19 October 2011

Academic Editor: Paolo Pennacchi

Copyright (๑) 2012 Ali Helali et al. This is an open access article distributed under the Creative Commons Attribution License, which permits unrestricted use, distribution, and reproduction in any medium, provided the original work is properly cited.

Presently, there exist few numerical methods which treat the inverse problem for the determination of the geometry of wind turbine blades. In this work, authors intend to solve the inverse optimum project for horizontal axis wind turbine in which the selection of the circulation distribution is obtained by resolving two variational problems: the first consists in sorting the circulation distribution on the lifting line, which, for a given power extracted by the wind turbine, minimizes the loses due to the induced velocity. In the second, the optimal circulation distribution is selected such that the kinetic energy of the wind downstream of the rotor disc is minimum, when the energy extracted by the wind turbine for one rotating period is imposed. A code has been developed which incorporates the real pitch of the helicoidal vortex wake. Very promising results have been obtained: the circulation distribution for a given extracted power and the chord lengths distribution law along the blade span.

\section{Introduction}

The blade element momentum (BEM) model [1] is the most common model used in aerodynamic and aeroelastic codes for the prediction of wind turbine characteristics. This is due to its simplicity, which makes it possible to implement the model as a fast numerical algorithm. The accuracy of the BEM model is in general reasonable for a wind turbine in normal operating conditions. A verification study [2] comprising the most common aeroelastic codes in Europe showed a typical difference of 5\%-10\% between measured and simulated mean blade loads and 5\%-20\% difference in dynamic blade loads. However, BEM simulations depend heavily on the input aerofoil sectional data, as was seen in the NREL blind test comparison of aerodynamic codes with data from the NASA-Ames wind tunnel experiment [1,3]. Usually a correction of the aerofoil data for three-dimensional flow effects is necessary in order to improve the correlation of numerical results with experimental data.

There are some fundamental shortcomings of the BEM model which can be ascribed to the assumptions introduced in its derivation. The equation relating the induction at the disc with the axial thrust is derived for a stream tube enclosing the whole rotor disc, but in the BEM model the same relation is used for differential stream tubes at different radial positions (strip theory). Comparison of the BEM model with more accurate induction models, such as the numerical actuator disc model with the flow solved with a CFD model, indicates that this assumption is not accurate in regions of the blade with strong radial variation of the loading, as, for example, towards the tip and at the root $[1,4]$.

Owing to the assumptions in the BEM model, more physically based aerodynamic models such as vortex line and panel methods have been developed for some problems (rotor shapes or flow conditions). These models reflect detailed information about the induced flow field at the rotor disc and have been successfully applied, for example, to studies of dynamic inflow situations $[1,5]$.

Though numerous studies have been effectuated on the resolution of the inverse problem on rotating machines, there exist few numerical methods which treat the inverse problem for the determination of the geometry of a wind turbine blade. 
If the wake of the blade and its slipstream were to be known exactly, then the field of induced velocities and other relevant quantities could be computed more readily. This can be done, in fact, by appropriate manipulation of Biot-Savart equation for the inviscid vortex line. The classical expression of a vortex-induced velocity is the Biot-Savart law that is the fundamental relationship between a vortex, its shape, and the velocity that it induces. This method proves extremely valuable, because it can track the main pattern of the vortex system.

The main idea of the present article consists in determining the circulation distribution which corresponds to a given extracted power. It is evident that the power coefficient should be chosen under Betz limit moreover under the limit curve for 3-blade Betz rotor [6]. We try to develop the inverse "OPHWT" model $[7,8]$. This model can provide us the optimal aerodynamic and geometric characteristics for a given extracted power. So after validating the "OPHWT" in [7], we try to exploit it really by using some of existing power coefficient for the machine.

The selection of the circulation distribution for the inverse problem is obtained by resolving two variational problems: the first consists in sorting the circulation distribution on the lifting line, which, for a given power extracted by the wind turbine, minimizes the loses due to the induced velocity "OPHWT" $[7,8]$. In the second, the optimal circulation distribution is selected such that the kinetic energy of the wind downstream of the rotor disc is minimum, when the energy extracted by the wind turbine for one rotating period is imposed.

It is shown that the optimal circulation distribution corresponding to the best energetic efficiency of the wind turbine induces a velocity which satisfies the slip condition. This actually occurs in the case where the helicoidally vortex sheet becomes rigid and rotates with the same speed as the wind turbine, but in the opposite sense.

The method used in calculating the induced velocities is publicized in [7].

The resolution is achieved by the singularities method based on discrete distributions of circulation: it consists in dividing the blade span-length $R$ in to a number of smallspanwise discrete segments of elementary lengths $\Delta r$, such that the divisions are progressively more concentrated towards the root and the tip and less concentrated towards the middle of the blade [7].

\section{Inverse OPHWT Model}

In this inverse "OPHWT" model, we intend in the reality to find the efficacy rotor. In fact, for a given wind site characterized by the wind speed and for a given number of the blades, we aim to use the machine with the minimum losses of power. These wishes consist in imposing some law for the induced velocities on the lifting line, and we determinate the optimum circulation distribution which induce these velocities.

The selection of the circulation distribution is based on the hypothesis of limiting the span length of the blade. An optimal circulation distribution is sorted by minimizing the power losses due to the effect of the induced velocity downstream of the turbine disc. It is a variational problem which consists in finding the circulation distribution $\Gamma(\xi)$ for which a power output $\bar{W}$ is as high as possible and which leads to a minimal losses $P$ due to the effect of the induced velocity $[7,8]$.

It should be noted that the wake shed along the blades is assumed to form a helicoidal vortex sheet originating from the trailing edge. The system of free vortices emanating from the trailing edge is specified to persist without disintegration up to a relatively far distance downstream of the rotor disc, by following a local stream line [9-12].

The method used in calculating the induced velocities by the vortex sheet is derived directly from the general lifting lines and lifting surfaces theories without any simplifications made. It was elaborated by Goldstein [9]. He was of the opinion that the rotation of the wake in the case of a finite number of blades constituted a loss of energy that would have to be provided by the propeller's motor and incorporated the loss in his theory. In order to correct for the losses, Goldstein used a complicated method based on the development of the circulation in terms of Bessel's functions and trigonometric series whose convergence is not assured especially towards the blades tips and the values of the circulation obtained are only poor approximations [7]. A recent development of these calculations was applied to marine screw propellers where the lifting line hypothesis was replaced by that of the lifting surface, because of the larger blades by Luu. The calculations of Morgan and Wrench [13] shall be adopted in the present paper. The vortex theory is applicable in the case of small attack angles where the lift curve of the blade sections is a linear function of the attack angle. The vortex sheet is as a result stationary with respect to the blades [7].

The integral equation for determining the optimum spanwise distribution of circulation $\Gamma(\xi)$ on the blade is given by [7]

$$
\begin{aligned}
& \int_{r_{m}}^{R} \Gamma_{M} \cdot \sum_{N_{P}} \int_{-\infty}^{+\infty} g(Q, M) \frac{r_{M}}{r_{Q}} \sqrt{\frac{V_{0}^{2}}{\Omega^{2}}+r_{Q}^{2}} d \alpha_{Q} d r_{M} \\
& \quad+\int_{r_{m}}^{R} \Gamma_{M} \cdot \sum_{N_{P}} \int_{-\infty}^{+\infty} g(M, Q) \sqrt{\frac{V_{0}^{2}}{\Omega^{2}}+r_{M}^{2}} d \alpha_{M} d r_{M}+C V_{0}=0 .
\end{aligned}
$$

A semi-infinite helicoidal vortex is made up of a vortex filament which is moving from the rotor disc plane to downstream infinity while carrying the vortex intensity $\left(\Gamma / \Omega R^{2}\right)_{j}$ (see Figure 1$)$. The component $(w / \Omega R)$ of the velocity induced according to Morgan and Wrench [13] is given by

$$
\begin{gathered}
\left(\frac{w}{\Omega R}\right)_{i}=\frac{N_{b}}{4 \pi \lambda^{*}}\left(1+G_{1}\right)\left(\frac{\Gamma}{\Omega R^{2}}\right)_{j} \text { if } \xi_{i} \prec \bar{\xi}_{j}, \\
\left(\frac{w}{\Omega R}\right)_{i}=-\frac{N_{b}}{4 \pi \lambda^{*}} G_{2}\left(\frac{\Gamma}{\Omega R^{2}}\right)_{j} \text { if } \xi_{i} \succ \bar{\xi}_{j},
\end{gathered}
$$




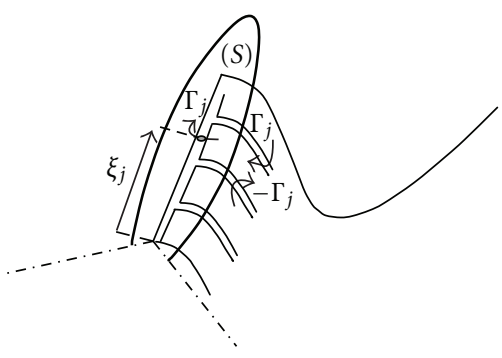

FIgURE 1: Helicoidal vortex sheet originating from the lifting line.

where $G_{1}$ and $G_{2}$ are given by

$$
\begin{gathered}
G_{1}=\left(\frac{z_{j}}{z_{i}}\right)^{1 / 2}\left[\frac{\mu}{1-\mu}-A \log (1-\mu)\right], \\
G_{2}=\left(\frac{z_{j}}{z_{i}}\right)^{1 / 2}\left[\frac{1}{\mu-1}+A \log \left(1-\mu^{-1}\right)\right] .
\end{gathered}
$$

With $y=\xi / \lambda^{*}, z=\sqrt{1+y^{2}}$ and

$$
\mu=\left(\frac{z_{i}-1}{y_{i}} \frac{y_{i}}{z_{j-1}}\right)^{N_{p}} e^{N_{p}\left(z_{i}-z_{j}\right)} .
$$

$A$ is given by Morgan and Wrench:

$$
A=\frac{1}{24 N_{p}}\left[\frac{9 y_{j}^{2}+2}{z_{j}^{3}}+\frac{3 y_{i}^{2}-2}{z_{i}^{3}}\right] .
$$

The power extracted by the wind turbine is written in the following form:

$$
\bar{W}=\int_{r_{m}}^{R} \rho \Omega r_{M}\left(V_{0}+w\right) \Gamma_{M} d r_{M}=W-P,
$$

where

$$
W=\int_{r_{m}}^{R} \rho \Omega r_{M} V_{0} \Gamma_{M} d r_{M}, \quad-P=\int_{r_{m}}^{R} \rho \Omega r_{M} w \Gamma_{M} d r_{M}
$$

$W$ represents the kinetic energy of wind (power extracted without any losses) and $P$ represents the losses resulting from the induced velocities.

The power coefficient $C_{P}$ is given by

$$
C_{P}=\frac{\int_{r_{m}}^{R} \rho \Omega r_{M}\left(V_{0}+w\right) \Gamma_{M} d r_{M}}{(1 / 2) \pi \rho R^{2} V_{0}^{3}} .
$$

Details of this model are presented in [7].

\section{Minimization of the Kinetic Energy Model}

The aim of this method is to minimize the kinetic energy of the wind down stream of the rotor disc. This leads to increasing the induced velocity downstream in order to attain the optimum efficiency which is under the limit curve for 3blade Betz rotor [6]. The optimization of wind turbines using helicoidal vortex model with minimization of the kinetic energy was made by Chattot in 2003 [14]. His solution differs from the present work because he used pure numerical simulations with a discretization of the helical vortex lines instead of the Morgan and Wrench's approximation. In this paper, numerical model is achieved by the singularities method based on discrete distributions of circulation: it consists in dividing the blade distance $r_{m} R$ in to a number small spanwise segments, such that the divisions are progressively more concentrated towards the root and the tip and less concentrated towards the middle of the blade $[6,7]$.

A variational calculation is used to show that the optimal distribution of the circulation $\Gamma(\xi)$ corresponding to a highenergy output by the wind turbine will induce a normal velocity $v_{n}$, whose expression is similar to that obtained by applying the slip condition, in the case where the helicoidal vortex sheet becomes stiff and turning with a rotational speed $\Omega \vec{u}_{z}$. The optimal condition is written as follows $[9,13]$ :

$$
v_{n}=\left(C \Omega \vec{u}_{z} \times r \vec{u}_{r}\right) \cdot \vec{n} .
$$

Indeed, if we designate by $\Sigma_{0}$ a portion of the helicoidal vortex sheet corresponding to one complete functioning period $T$, the kinetic energy $E$ imparted to the fluid downstream of the rotor disc during the period is given by

$$
E(\Gamma)=-\frac{\rho}{2} \int_{\Sigma_{0}} \Gamma \phi_{n}^{\prime} d \Sigma .
$$

The power extracted by the wind turbine for one functioning period is given by

$$
\begin{aligned}
U(\Gamma) & =\int_{r_{m}}^{R} \int_{0}^{T} \rho\left(\Omega \vec{u}_{z} \times r \vec{u}_{r}\right) \cdot\left(\Gamma d r \vec{u}_{r} \times W d t \vec{u}_{s}\right) d \Sigma \\
& =\rho \Omega \int_{\Sigma_{0}} r \vec{u}_{\theta} \cdot \vec{u}_{n} \Gamma d r .
\end{aligned}
$$

The target now is to look for the condition to be satisfied so that the kinetic energy $E$ is minimal when the value of $U$ in the preceding (5) is imposed. Let us suppose that there is a variation of the circulation distribution $\Gamma(r)$; by designating this variation by $\delta \Gamma(r)$, the variation of $U$ which results from (5) is given by

$$
\delta U(\delta \Gamma)=\rho \int_{\Sigma_{0}} \Omega r \vec{u}_{\theta} \cdot \vec{u}_{n} \delta \Gamma \delta \Sigma
$$

The corresponding variation of $E$ is given by

$$
\delta E(\delta \Gamma)=-\frac{\rho}{2} \int_{\Sigma_{0}}\left(\delta \Gamma \phi_{n}^{\prime}+\Gamma \delta \phi_{n}^{\prime}\right) \delta \Sigma .
$$

Now the velocity $\phi_{n}^{\prime}$ induced by a doublet distribution of intensity $\Gamma$ into the points $P$ and $M$ on the sheet $\Sigma_{0}$ is given by

$$
\begin{aligned}
\left(\phi_{n}^{\prime}\right)_{M} & =\int_{\Sigma_{0}} \Gamma(P) \frac{\partial}{\partial n_{M}} \frac{\partial}{\partial n_{P}} g(P, M) d \Sigma_{P} \\
& =\int_{\Sigma_{0}} \Gamma(P) k(P, M) d \Sigma_{P},
\end{aligned}
$$


where $g(P, M)=1 /\left(4 \pi r_{P M}\right)$ and the function $k(P, M)$ is defined by:

$$
k(P, M)=\frac{\partial}{\partial n_{M}} \frac{\partial}{\partial n_{P}} g(P, M),
$$

$k(P, M)$ is a symmetric function with respect to the variables $P$ and $M$; as a result we can write

$$
\begin{gathered}
\delta E(\delta \Gamma)=-\frac{\rho}{2} \int_{\Sigma_{0}}(\delta \Gamma \Gamma k(P, M)+\Gamma \delta \Gamma k(P, M)) \delta \Sigma_{P}, \\
\delta E(\delta \Gamma)=-\rho \int_{\Sigma_{0}} \partial \Gamma \phi_{n}^{\prime} d \Sigma_{P} .
\end{gathered}
$$

Let $\partial \Gamma$ and $\partial \Gamma^{\prime}$ be arbitrary variations of the initial distribution of $\Gamma(P)$; the corresponding variations of $U$ are $\partial U(\partial \Gamma)$ and $\partial U\left(\partial \Gamma^{\prime}\right)$. The two variations of $\Gamma$ can be superposed in such a way that the resulting variation of $U$ is zero. This implies that $U$ remains unchanged as expressed in the following equation:

$$
\partial U(\partial \Gamma)-k \partial U\left(\partial \Gamma^{\prime}\right)=0 .
$$

The resulting variation of $\Gamma(P)$ is $\partial \Gamma-k \partial \Gamma^{\prime}$.

The corresponding variation of $E$ is equally given by $\partial E(\partial \Gamma)-k \partial E\left(\partial \Gamma^{\prime}\right)$.

If $E(\Gamma)$ corresponding to the initial distribution of $\Gamma$ is extremum; that is, it is either minimal or maximal, then the corresponding variation of $E$ has to be zero. This last condition enables us to obtain an expression for $k$ given by $k=$ $\partial E(\partial \Gamma) / \partial E\left(\partial \Gamma^{\prime}\right)$

By substituting $k$ in (10), we obtain

$$
\partial U(\partial \Gamma)-\frac{\partial U\left(\partial \Gamma^{\prime}\right)}{\partial E\left(\partial \Gamma^{\prime}\right)} \partial E(\partial \Gamma)=0 .
$$

By introducing the Lagrange constant: $C=\left[\partial U\left(\partial \Gamma^{\prime}\right)\right] /$ $\left[\partial E\left(\partial \Gamma^{\prime}\right)\right]$, the preceding equation becomes: $\partial U(\partial \Gamma)-$ $C \partial E(\partial \Gamma)=0$.

By considering the expressions which give $\partial U(\partial \Gamma)$ and $\partial E(\partial \Gamma)$, that is, (6) and (9), the optimal condition is written as

$$
\rho \int_{\Sigma_{0}}\left(\Omega r \vec{u}_{\theta} \cdot \vec{u}_{n}-C \phi_{n}^{\prime}\right) \partial \Gamma d \Sigma_{P}=0 .
$$

We notice that this is a homogenous equation. If $\partial \Gamma$ must be different from zero, then the content of the bracket must be zero; this leads to

$$
C \phi_{n}^{\prime}=\Omega r \vec{u}_{\theta} \cdot \vec{u}_{n}
$$

in which $\left(\phi_{n}^{\prime}\right)$ is recognized as a speed $v_{n}$ resembling the slip condition in the case of a stiff helicoidal vortex sheet rotating at a suitable angular velocity. The optimal condition in (3) is written now as

$$
v_{n}=C \Omega r \vec{u}_{\theta} \cdot \vec{n} .
$$

But the component in the direction of $\vec{u}_{\theta}$ of the vector normal to the vortex sheet is given by

$$
\vec{u}_{\theta} \cdot \vec{n}=n_{\theta}=\frac{\lambda^{*}}{\left(\lambda^{*}+\xi^{2}\right)^{1 / 2}},
$$

where $\lambda^{*}$ is the parameter characterising the real advance pitch of the vortex system.

Hence, the normal component of the induced velocity corresponding to the optimal condition is given by

$$
v_{n}=\frac{C \lambda^{*} \Omega r}{\left(\lambda^{2}+\xi^{2}\right)^{1 / 2}} .
$$

Now, the normal component of the induced velocity is linked to the axial component $v_{z}$, along $\vec{u}_{z}$ by

$$
v_{n}=\frac{1}{\xi}\left(\lambda^{*^{2}}+\xi^{2}\right)^{1 / 2} v_{z} .
$$

It comes out that the optimal condition concerning the component $v_{z}$ of the induced velocity is given by

$$
\frac{v_{z}}{\Omega R^{2}}=\frac{C \lambda^{*} \Omega \xi^{2}}{\lambda^{* 2}+\xi^{2}} .
$$

The optimal distribution of $\Gamma^{*}(\xi)$ is obtained to a multiplicative constant by resolving the following linear system of equations deduced from a combination of the Biot Savart's law and the calculations of Morgan and Wrench [13]:

$$
\sum_{j=1}^{j=11}(\mathrm{VZIJ})_{i, j}\left(\frac{\Gamma^{*}}{\Omega R^{2}}\right)_{j}=\frac{\xi^{2}}{\lambda^{*^{2}}+\xi^{2}},
$$

where $\Gamma^{*}(\xi)=\Gamma(\xi) / \lambda^{*}$.

The resolution of (20) is achieved by the singularities method based on discrete distributions of circulation. The initial values of the circulation distributions and the components of the induced velocities obtained can be further improved iteratively by introducing a multiplicative factor $X$, calculated by maximizing the torque coefficient. See $[7$, appendix 3]. Details of these calculations are presented in [7].

\section{Results and Discussions}

For a given extracted power, we present in Figures 2 and 3 the optimal circulation coefficient for the two models. In fact, for a given wind site characterized by the wind speed to tip speed ratio at the extremity of the blade (lambda: $\lambda_{0}=V_{0} / \Omega R$ ) and for a given number of the blades $\left(N_{b}=3\right)$, we determine the optimum circulation distribution which corresponds to the maximum power extractable by the wind turbine.

This result is very important in designing the blade of a wind turbine. In effect, by using this code we can determine the optimum circulation for every given power coefficient; later on we can determine the chord lengths distribution along the blade span leading to the optimum shape of the blade. This is the main the advantage of the OPHWT model.

A comparison of the next two figures reveals some discrepancy between the two models. This is presented in Figure 4. The circulation coefficient distribution of the OPHWT model appears to be quasiconstant on the lifting line. But for the kinetic energy model this distribution gives the impression to be an elliptic function. It is further seen that 


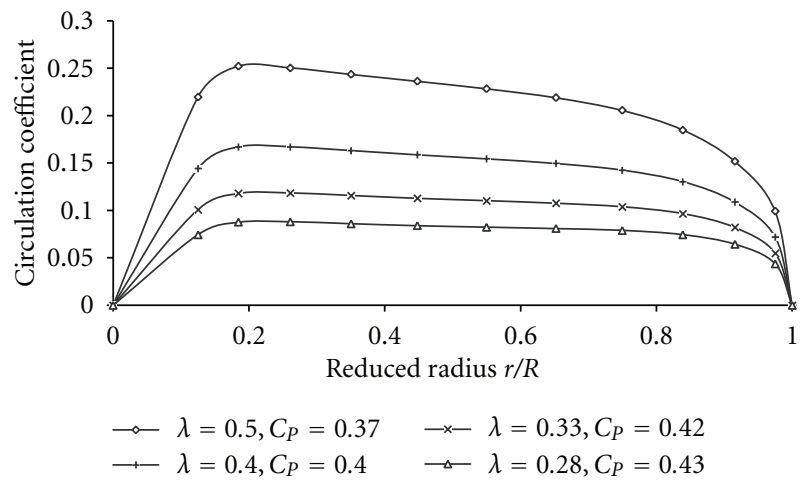

Figure 2: Circulation Coefficient on the lifting line for $N_{b}=3$ for the inverse OPHWT model.

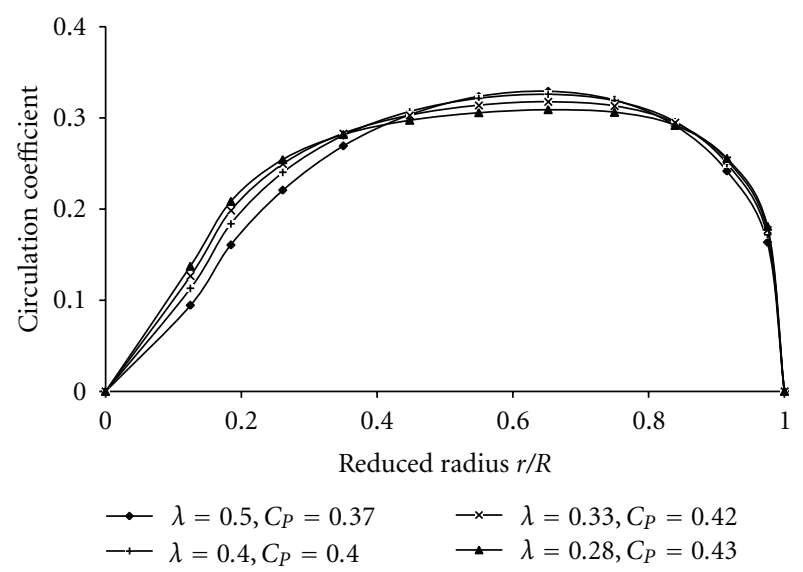

FIgure 3: Circulation Coefficient on the lifting line for $N_{b}=3$ obtained by the kinetic energy model.

the circulation for the two models goes to zero at both ends of the blade, which is well known from finite aspect ratio wing theory.

For the same coefficient power $C_{p}=0.37$, the difference in the value notified in Figure 4 reaches 30\% in the reference chord spanwise as defined at $0.75 R$. This can be attributed to the higher induced velocity in the case of the model where the kinetic energy of the wind downstream of the rotor disc is minimized, and for the reason that in the OPHWT model we reduce the effect of the normal component of the induced velocity.

Figures 5 and 6 present the variation of the chord lengths of the blade sections along the span for an incident angle of $8^{\circ}$ for the two models for a fixed power $C_{p}$. We make some variations on the power coefficient with the functioning parameter lambda $\left(\lambda_{0}=V_{0} / \Omega R\right)$ for a wind turbine of 3 blades. This point is perfectly intended by the constructers who suggest to manufacture the specific wind turbine for a given wind site.

Figure 6 reveals clearly the most resistant form of a blade characterized by larger chords towards the hub which progressively becomes less large towards the tip in order to maintain the quasiconstant circulation along the blade (see

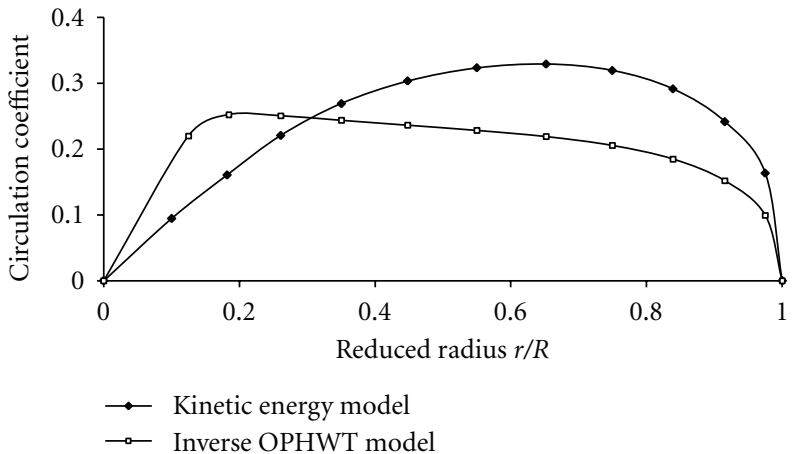

FIGURE 4: Comparison of the circulation coefficient distribution on the lifting line between the OPHWT model and the kinetic energy model for $\lambda_{0}=0.5$ and $C_{P}=0.37$.

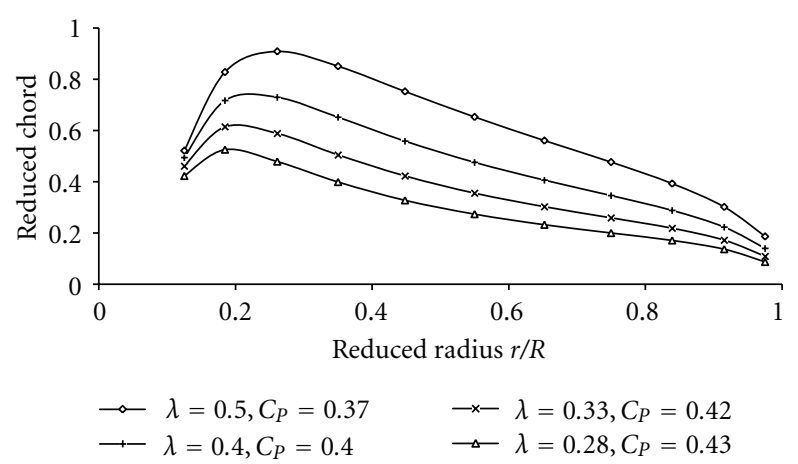

FIGURE 5: Spanwise variation of the chord in the inverse OPHWT model for $N_{b}=3$ and $\alpha=8^{\circ}$.

Figure 2) and resulting in the possibility of the blades enhancing higher loads. It is the well-known shape of the blade of the wind turbine.

However for the same functioning parameter $\lambda_{0}$ (lambda), those predicted by the optimum project are larger because of the hypothesis of minimization of the losses due to the induced velocity. In effect, the OPHWT model is applicable to the wind turbines while the minimization of the kinetic energy is used for the propellers of highest efficiency in which the motive power supplies the torque which is necessary to maintain the rotation. The chord in the case of the kinetic energy model has an elliptic form (Figure 6) which bears a resemblance to the parabolic shape turning on the highest velocities.

The OPHWT is confirmed very efficient to calculate the optimal performances and geometry of a wind turbine. In effect, it is applied in the case of reel functioning conditions. Figure 7 presents the circulation distribution of the OPHWT model compared to that of the near wake model of Beddoes $[1,15]$ for a $40 \mathrm{~m}$ blade rotating at a free wind speed of $7 \mathrm{~ms}^{-1}$ and for a coefficient power $C_{p}=0,48$. The corresponding distribution of the plan-form is shown in Figure 8.

As can be observed in Figure 7, the circulation distributions follow the same trend but there are some discrepancies in the values of the circulation distributions at the tip of 


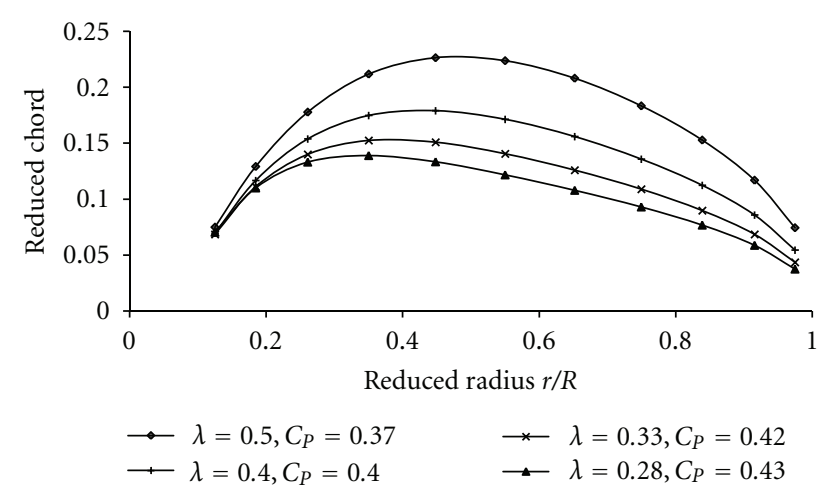

FIGURE 6: Span wise variation of the chord in the kinetic energy model for $N_{b}=3$ and $\alpha=8^{\circ}$.

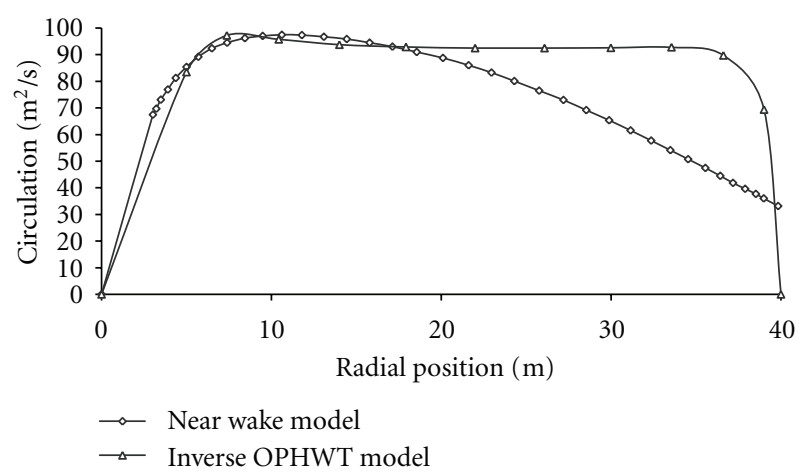

Figure 7: Comparison of the circulation $\Gamma\left(\mathrm{m}^{2} / \mathrm{s}\right)$ between the inverse OPHWT model and the near wake model for the $40 \mathrm{~m}$ rotating blade at a free wind speed of $7 \mathrm{~ms}^{-1}$ and for $C_{p}=0.48$.

the blade. This divergence attains $29 \%$ at the section with abscissa $30 \mathrm{~m}$ on the blade. In effect, the OPHWT model is based on a variational problem aiming at reducing the energy losses due to the induced velocity by the vortex sheet. The near wake model on the other hand is in principle a lifting line model for the rotating blade, where only a quarter revolution of the wake system behind the blade is taken into account. In this model, Beddoes [15] considers that only the first part of the trailed vortex system behind the blade has an important influence on the shaping of the loading on the blade as well as on the time variation of the downwash at the blade. The circulation distribution of the OPHWT model is thus believed to be the most closest to reality because this circulation seems to be quasiconstant on the lifting line except towards the root and at the tip of the blade where it falls to zero.

The Near wake model was tested on a $40 \mathrm{~m}$ rotating blade with an angular velocity of $2.0 \mathrm{rad} \mathrm{s}^{-1}$ corresponding to a tip speed of $80 \mathrm{~m} \mathrm{~s}^{-1}$ and with a plan-form as shown in Figure 8. The plan-form of the Near wake model is compared to the plan-form obtained by the inverse OPHWT model. The spanwise variations of the chords follow the same trend but there are discrepancies in the values at the bottom of the blade. In effect, the chords in the present inverse problem correspond to a power coefficient of 0.48 obtained by using

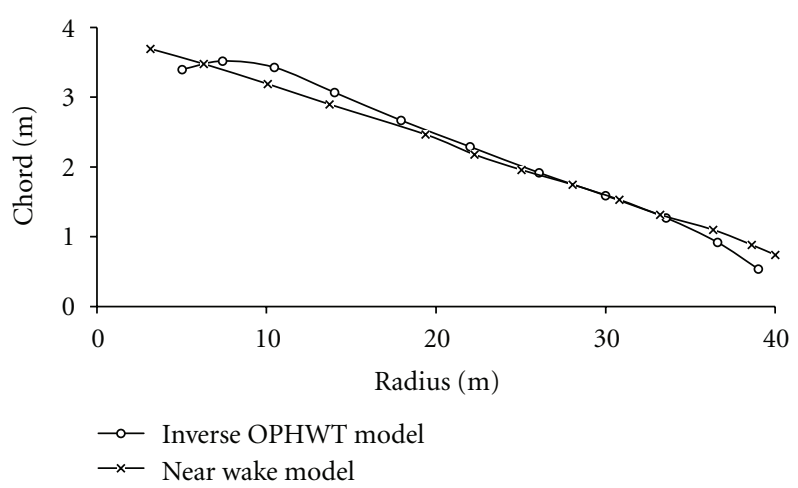

FIGURE 8: Spanwise variation of the chord in the inverse OPHWT model and the Near wake model for $N_{b}=3$ and $\alpha=8^{\circ}$.

the circulation equation $\Gamma=k_{\infty} C(r) \vec{W}(r) \alpha(r)$. But for the near wake model, the plan-form presented in Figure 8 is an input used to determine the circulation distribution presented in Figure 7.

\section{Conclusion}

In this paper, an inverse problem has been resolved by two methods: the first consists in the minimization of the power losses due to the induced velocity (inverse OPHWT model) and in the second we have minimized the kinetic energy imparted to the fluid downstream of the rotor disc (prop fan).

Confrontation of the results obtained with that of Helge Aaggard and Flemming (A near wake model for Beddoes) has revealed the potential of the optimum project in predicting improved and higher rotor performances even at relatively high wind speeds.

In effect, for a given wind site characterized by a windrose, that is a given functioning parameter $\lambda_{0}$, a fixed power coefficient $C_{P}$ is required for the wind turbine. The inverse OPHWT code can provide us with the optimal circulation distribution as well as the most adequate plan-form for the construction of the blade corresponding to the given $C_{P}$.

As a follow-up of this work we intend to construct a complete blade of a wind turbine in 3D by coupling the inverse OPHWT model with an inverse code which solves the inverse problem of the flow over a 2D airfoil $[16,17]$.

The aim of the project is to visualize the actual level of wind turbine technology in Tunisia (the Sidi Daoud wind site, Tunisia): essentially the blades and the aerodynamic efficiency in order to set up a local wind turbine industry. Tunisia intends to install other wind sites in order to reach an energy production of about $200 \mathrm{MW}$ by 2012. This paper can help the decision makers in the choice of the blades suitable for a given wind site, which can be purchased, and also to design new forms of blades for the small installations of wind turbines for isolated sites.

\section{References}

[1] M. Helge Aaggard and R. A. Flemming, "A near wake model for trailing vorticity compared with the blade element momentum theory," Wind Energy, vol. 7, no. 4, pp. 325-341, 2004. 
[2] J. G. Schepers et al., "Verification of European wind turbine design codes," VEWTDC; Final Report. ECN-C-OI-055, 2002.

[3] D. Simms, S. Schreck, M. Hand, and L. J. Fingersh, "NREL Unsteady Aerodynarnics Experiment in the NASA-Ames wind tunnel: a comparison of predictions to measurements," Report NREl/TP-500-29494, 2001.

[4] H. A. Madsen, "A CFD analysis of the actuator disc flow compared with momentum theory results," in Proceedings of the 10th Symposium on Aerodynamics of Wind Turbines, pp. 109124, IEA Joint Action, Edinburgh, Germany, 1996.

[5] H. Snel, "Survey of induction dynarnics modelling within BEM-like codes," AlAA Paper 2001-0027, 2001.

[6] V. L. Okulov and J. N. Soørensen, "Maximum efficiency of wind turbine rotors using Joukowsky and Betz approaches," Journal of Fluid Mechanics, vol. 649, pp. 497-508, 2010.

[7] B. Kamoun, A. Helali, and D. Afungchui, "Optimum project for horizontal axis wind turbines OPHWT," Renewable Energy, vol. 30, no. 13, pp. 2019-2043, 2005.

[8] A. Helali, D. Afungchui, and B. Kamoun, "Characteristics of the Sidi Daoud (Tunisia) wind farm site and the optimal aerodynamic modelling of a wind turbine," in Proceedings of the International Congress on the Renewable Energies and the Environment (CERE '05), Sousse, Tunisia, March 2005.

[9] S. Goldstein, "On the vortex theory of screw propeller," Royal Society Proceedings A, vol. 123, no. 440, 1929.

[10] A. A. Afjeh and T. G. Keith Jr., "A vortex lifting line method for the analysis of horizontal axis wind turbines," Journal of Solar Energy Engineering, vol. 108, no. 4, pp. 303-309, 1986.

[11] D. Sharpe, "A lifting line theory for the determination of wind turbine blade optimum performance," in Proceedings of the European Wind Energy Conference, Madrid, Spain, 2003.

[12] J. M. Bousquet, Introduction à L'aérodynamique des Hélices, Ecole Supérieure des Techniques Aérospatiales (ESTA), Paris, France, 1998.

[13] W. B. Morgan and J. W. Wrench, "Some computational aspects of propeller design," in Methods in Computational Physics, vol. 4, pp. 301-331, Academic Press, New York, NY, USA, 1965.

[14] J. J. Chattot, "Optimization of wind turbines using helicoidal vortex model," Journal of Solar Energy Engineering, Transactions of the ASME, vol. 125, no. 4, pp. 418-424, 2003.

[15] T. S. Beddoes, A near wake dynamic model. Aerodynamics and Aeroacoustics National Specialist Meeting. Papers and Discussion, 1987.

[16] B. Kamoun, D. Afungchui, and A. Chauvin, "A wind turbine blade profile analysis code based on the singularities method," Renewable Energy, vol. 30, no. 3, pp. 339-352, 2005.

[17] B. Kamoun, D. Afungchui, and M. Abid, "The inverse design of the wind turbine blade sections by the singularities method," Renewable Energy, vol. 31, no. 13, pp. 2091-2107, 2006. 

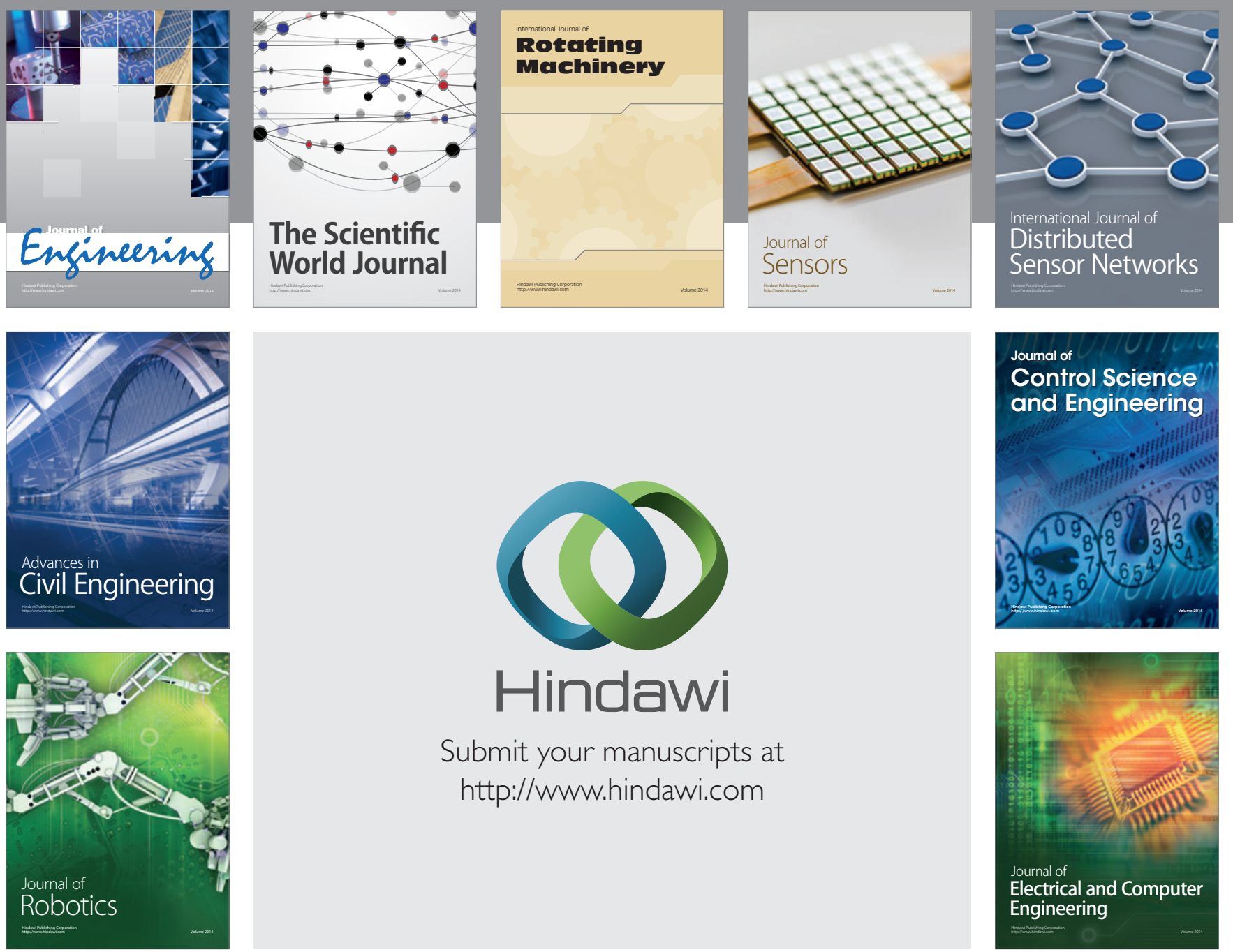

Submit your manuscripts at

http://www.hindawi.com
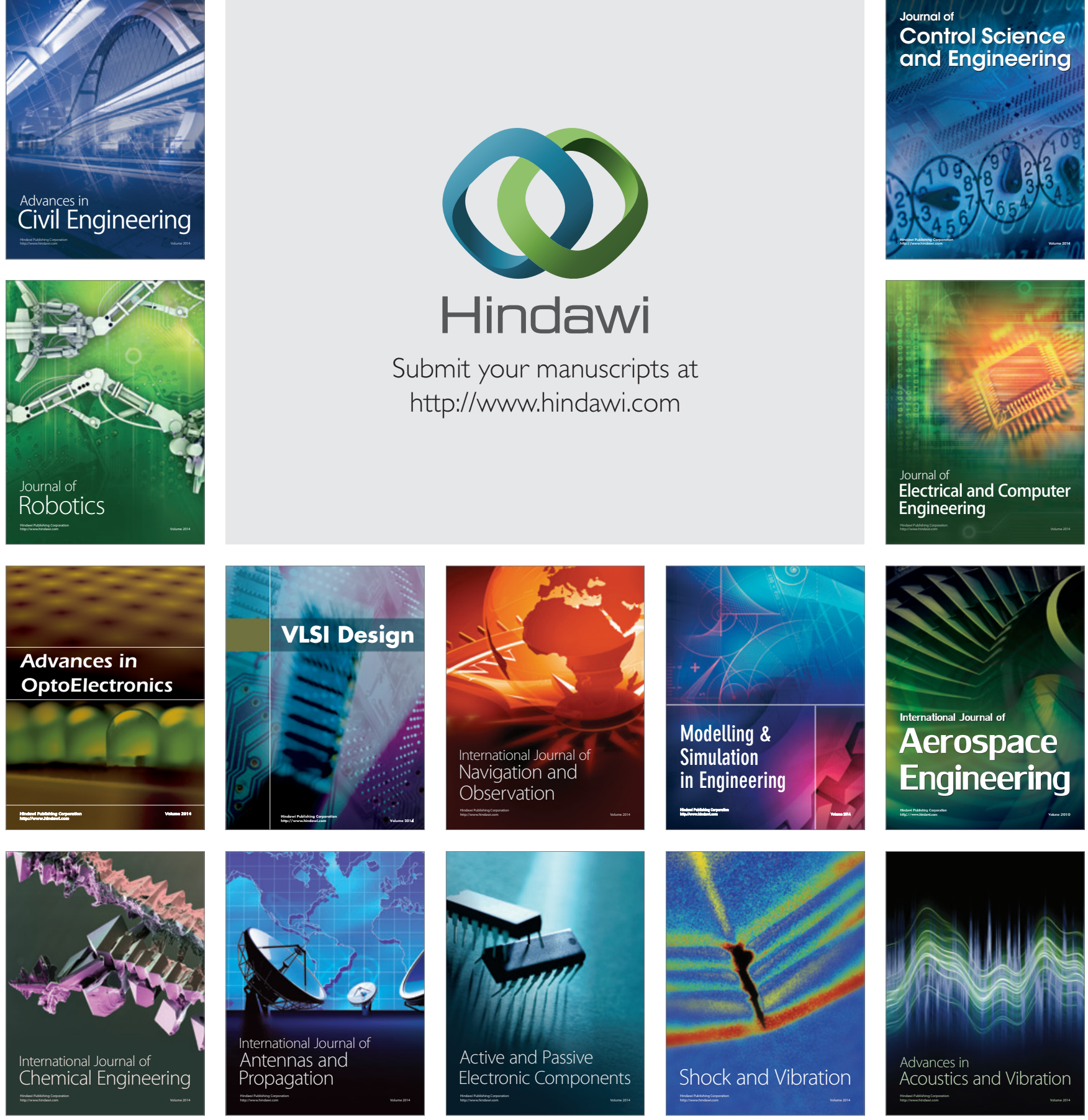